\title{
The Use of Subgroup Disproportionality Analyses to Explore the Sensitivity of a Global Database of Individual Case Safety Reports to Known Pharmacogenomic Risk Variants Common in Japan
}

\author{
Rika Wakao $^{1}$ (D) $\cdot$ Ingrid M. Lönnstedt ${ }^{2} \cdot$ Yasunori Aoki $^{2,3}$ (D) $\cdot$ Rebecca E. Chandler $^{2}$
}

Accepted: 7 March 2021 / Published online: 10 April 2021

(c) The Author(s) 2021

\begin{abstract}
Introduction Genetic variations of enzymes that affect the pharmacokinetics and hence effects of medications differ between ethnicities, resulting in variation in the risk of adverse drug reactions (ADR) between different populations. Previous work has demonstrated that risk-group considerations can be incorporated into approaches of statistical signal detection. It is unknown whether databases of individual case safety reports (ICSRs) are sensitive to pharmacogenomic differences between populations.

Objective The aim of this study was to explore the sensitivity of a global database of ICSRs to known pharmacogenomic risk variants common in Japan.

Methods The data source was VigiBase, the global database of ICSRs, including all reports entered in the version frozen on 5 January 2020. Subgroup disproportionality analysis was used to compare ICSRs of two subgroups, Japan and rest of world (RoW). Reports for UGT1A1-metabolized irinotecan and the CYP2C19-metabolized drugs voriconazole, escitalopram and clopidogrel were selected for comparison between the subgroups based upon known genetic polymorphisms with high prevalence in Japan. Contrast between the subgroups was quantified by IC delta $\left(\mathrm{IC}_{L}\right)$, a robust shrinkage observed-toexpected (OE) ratio on a log scale. Harmonic mean $p$ values (HMP) were calculated for each drug to evaluate whether a list of pre-specified ADRs were collectively significantly over- (or under-)reported as hypothesized. Daily drug dosages were calculated for ICSRs with sufficient information, and dose distributions were compared between Japan and RoW and related to differences in regionally approved doses.

Results The predictions of over-reporting patterns for specific ADRs were observed and confirmed in bootstrap HMP analyses ( $p=0.004$ for irinotecan and $p<0.001$ for each of voriconazole, escitalopram and clopidogrel) and compared with similar drugs with different metabolic pathways. The impact of proactive regulatory action, such as recommended dosing and therapeutic drug monitoring (TDM), was also observable within the global database. For irinotecan and escitalopram, there was evidence of use of lower dosages as recommended in the Japanese labels; for voriconazole, there was evidence of use of TDM with an over-reporting of terms related to drug level measurements and an under-reporting of liver toxicity. Conclusions Pharmaco-ethnic vulnerabilities caused by pharmacogenomic differences between populations may contribute to differences in ADR reporting between countries in a global database of ICSRs. Regional analyses within a global database can inform on the effectiveness of local risk minimization measures and should be leveraged to catalyse the conversion of real-world usage into safer use of drugs in ethnically tailored ways.
\end{abstract}

\section{Introduction}

Rebecca E. Chandler

Rebecca.Chandler@who-umc.org

1 Pharmaceuticals and Medical Devices Agency (PMDA), Tokyo, Japan

2 Uppsala Monitoring Centre, Box 1051, 75140 Uppsala, Sweden

3 National Institute of Informatics, Tokyo, Japan
Databases of individual case safety reports (ICSRs) of suspected adverse drug reactions (ADRs) are the cornerstone of pharmacovigilance. Designed for the purpose of hypothesis generation, these databases are used for statistical screening in the detection of signals of harm caused by medicines. However, as their content has grown and evolved over 


\section{Key Points}

Pharmacogenomics can explain variations in the susceptibility to adverse drug reactions (ADRs) between different populations.

Using subgroup disproportionality analyses, pharmacoethnic differences in pharmacokinetics prevalent in the Japanese population are visible in a global database of suspected ADRs.

Data contained within databases of individual case safety reports should be further explored to identify populationspecific safety issues and inform on the effectiveness of pharmacogenomic-directed risk mitigation measures in a region.

decades, the data contained within such databases may be useful beyond the identification of new potential causal relationships between drugs and adverse events. Recent work from the Uppsala Monitoring Centre has demonstrated that risk-group considerations can be implemented into statistical screening of databases of ICSRs using subgroup disproportionality analyses [1].

Analyses within VigiBase, the global database of the WHO Program of International Drug Monitoring, provide the opportunity to explore regional or country data in a global context. A previous study of Japanese adverse event reporting patterns revealed key features of Japanese reports suggestive of differences in adverse event reporting and/or clinical practice as well as possible pharmaco-ethnic vulnerabilities in the Japanese in contrast with Caucasians, the ethnicity representing the largest proportion of rest of the world (RoW) reports in VigiBase [2]. The frequency of genetic variations of enzymes that affect the pharmacokinetics and hence effects of medications differ between ethnicities, resulting in variation in the susceptibility to or risk of ADRs between different populations. It is unknown whether databases of ICSRs reflect expected pharmacogenomic differences between populations, but a comparison of reports from Japan versus those from the RoW within VigiBase would provide a model case to explore this question.

Uridine diphosphate glucuronosyl transferase (UGT) 1A1 and cytochrome P450 (CYP) 2C19 are enzymes with well characterized genetic polymorphisms and distinct subgroups within the Japanese population [3, 4]. Genetic polymorphisms of the UGT1A1 gene explain the variability of irinotecan-related toxicity among patients. The UGT1A1*28 (rs8175347) polymorphism is a known biomarker of irinotecan-induced neutropenia with minor allele frequency of $29-45 \%$ in Caucasians and only $16 \%$ in Asian populations, while UGT1A1 *6 (rs4148323) is the most frequent and important polymorphism among the East Asian population with a frequency of $15-20 \%$ [3]. The UGT1A $1 * 28$ allele is not considered a major risk factor for toxicity for low-dose use of irinotecan according to European guidance [5], but there is evidence that the presence of the UGT1A $1 * 6$ allele predisposes to neutropenia and to a lesser extent to diarrhoea at low doses in the Japanese population [3].

The wild-type CYP2C19*1 allele is associated with functional CYP2C19-mediated metabolism. In Europeans and Africans, the *17 (rs12248560) promoter variant with increased CYP2C19 activity is most abundant, accounting for $42-55 \%$ of all variant alleles, whereas the $* 2$ (rs4244285) loss-of-function variant is most prevalent in Asian populations, accounting for $78.5 \%$ of all variant alleles in East Asians. Another major CYP2C19 loss-of-function variant, CYP2C19*3 (rs4986893), is abundant exclusively in East Asians (minor allele frequency $=6.7 \%$ ). As expected functional consequences of allelic distributions, Europeans have $22.4 \%$ increased, $59.1 \%$ normal and $18.5 \%$ decreased functional activity, while East Asians have $1.5 \%$ increased, $60.5 \%$ normal and $38 \%$ decreased activity [4]. Accordingly, the CYP2C19-metabolized drugs have a greater inherent risk to Asian people, and many drugs, such as voriconazole, escitalopram and clopidogrel, have CYP2C19-related information (poor, intermediate, normal and rapid metabolizers) highlighted in the Japanese product labels [6-8]. Voriconazole and escitalopram require CYP2C19 for their metabolism to inactive forms, while clopidogrel is a prodrug and requires CYP2C19 for activation to its active form.

The aim of this study was to explore the sensitivity of a global database of ICSRs to known pharmacogenomic risk variants common in Japan. Our hypothesis was that subgroup disproportionality analyses would reveal a higher level of reporting of certain ADRs for irinotecan, voriconazole, escitalopram and clopidogrel from Japan compared with the RoW, given the inherent risk to the Japanese due to known genotypes of UGT1A1 and CYP2C19.

\section{Methods}

\subsection{Dataset}

The source of data was VigiBase, the WHO global database of ICSRs, which includes reports of adverse events shared by national pharmacovigilance centres in the member countries of the WHO Programme for International Drug Monitoring [9]. The reports in VigiBase come from a variety of sources, including physicians, pharmacists and consumers, are mostly spontaneously submitted or, less commonly, come from studies, and-importantly-indicate varying degrees of 
probability that the adverse event is drug related. VigiBase is maintained by the Uppsala Monitoring Centre (UMC).

Analyses were performed on the active ingredient level of drugs, coded according to WHODrug Global [10], and on the Preferred Term (PT) level of adverse events, coded according to MedDRA ${ }^{\circledR}$, the Medical Dictionary for Regulatory Activities terminology (version 20.0) [11].

The dataset used for this study consisted of all reports entered in VigiBase in the version frozen on 5 January 2020 at 1400 hours Central European Time (CET). Suspected duplicate reports were identified with the vigiMatch algorithm [12] and excluded from the dataset. Case reports were included in the analysis if they fulfilled the following:

- Classified as serious by the reporter.

- Patient domestic to the country of the report, as judged by UMC.

- MedDRA PT validated by UMC (to avoid reports with no MedDRA reaction).

- Drug characterized by UMC (to avoid reports lacking drug content).

All statistical analyses were performed with the software $\mathrm{R}$, version 4.0.0 [13].

\subsection{Statistical Methods}

Subgroup disproportionality analysis, as described by Hopstadius and Norén [14], was used as the basis for this study. Two subgroups were defined within this study, Japan and RoW. Contrast between the subgroups was quantified by IC delta $\left(\mathrm{IC}_{L}\right)$, a robust shrinkage observed-to-expected (OE) ratio on a $\log$ scale. A positive $\mathrm{IC}_{\Delta}$ therefore means more reports were seen in Japan than expected if the rate of reporting were the same in Japan as in the RoW. Similarly, a negative $\mathrm{IC}_{\Delta}$ means fewer reports were seen in Japan than expected. A positive (negative) $\mathrm{IC}_{\Delta}$ was taken to reflect over(under-)reporting in Japan if significant on the 5\% significance level.

For each of our four drugs under investigation (irinotecan, voriconazole, escitalopram and clopidogrel), we specified ADRs expected to be over-reported (or under-reported for clopidogrel) in Japan compared with RoW based upon predictions from known differences in prevalence of pharmacogenomic variants of UGT1A1 and CYP2C19 between Japan and RoW.

To evaluate whether the list of $R$ pre-specified ADRs of one drug collectively expressed significant over- (or under-) reporting as hypothesized, a harmonic mean $p$ value (HMP) was calculated for the drug, based on all the $R$ single ADR $p$ values in the pre-defined ADR set. This HMP was compared with 1000 bootstrap HMP values, each calculated from a random set of $R$ ADRs selected from the set of all ADRs reported for the drug in the RoW, giving an empiric bootstrap $p$ value for the drug. This $p$ value reflects the omnibus over- (or under-) reporting of the pre-specified ADRs for the drug.

For each of our four investigated drugs, one or two drugs with the same mechanism of action but not relying on the same drug metabolism were chosen for comparison. The $I C_{\Delta}$ and the HPM of the pre-specified ADRs were calculated for the comparator drugs and compared with the results of the investigated drug (e.g. Fig. 1a for irinotecan) in order to evaluate the hypothesis of pharmacogenomics being visible in the reporting pattern of the investigated drug.

As a descriptive summary of over-reported ADRs, we also provide a treemap for each of the four drugs (e.g. Fig. $1 \mathrm{~b}$ for irinotecan). The treemaps display each statistically significantly over-reported ADR (in Japan compared with RoW) in a box with the area proportional to the frequency of reports on that drug-ADR combination in Japan and with the colour representing $\mathrm{IC}_{\Delta}$-the higher the $\mathrm{IC}_{\Delta}$, the more over-reporting in Japan. The ADRs in the treemaps are displayed in groups by System Organ Class (SOC), so we can see which SOC is most common by comparing SOC areas. Full details of statistical analyses are available in the electronic supplementary material (ESM). Abbreviations for the individual systems within SOCs that are used in all figures are presented in Supplementary Table 1 (see ESM).

The dose distributions of each drug were graphed as histograms for comparison between Japan and RoW. Daily dosages are calculated for adult patient ICSRs when reported as a dose weight in $\mathrm{g}$, $\mathrm{mg}$ or $\mathrm{mg}$ together with a dose number and the interval at which the number of dose units were taken (per day, per hour or in total). This information is available for 8\% of ICSRs in VigiBase and was available for 112,539 cases extracted for this analysis.

\subsection{Rationale for Selected Comparisons}

The drugs selected for comparison were irinotecan and topotecan; voriconazole and fluconazole and fosfluconazole; escitalopram and sertraline and milnacipran; clopidogrel and prasugrel.

Irinotecan is an antineoplastic agent that acts as a specific inhibitor of type I DNA topoisomerase. The major dose-limiting toxicities (DLTs) in irinotecan chemotherapy are myelosuppression (neutropenia and leukopenia) and delayed-onset diarrhoea [15]. Accordingly, we preselected the MedDRA PTs including myelosuppression, diarrhoea and other terms designating bone marrow depression or diarrhoea (Supplementary Table 2, see ESM). Topotecan was chosen as a comparator since it is the same class of topoisomerase I inhibitor as irinotecan, but it is not metabolized by UGT; it undergoes a reversible $\mathrm{pH}$-dependent hydrolysis of its lactone moiety to its active form and is metabolized 
Fig. 1 Irinotecan. a HMP analysis of pre-specified PTs for irinotecan and topotecan. Drug-specific ADRs were selected prior to the study as suspected for over-reporting in Japan compared with RoW if the hypothesis of increased risk to Japanese were true. An HMP $<0.05$ indicates that the $\mathrm{IC}_{\Delta} s$ of the preselected PTs are collectively significantly over-reported, supporting the hypothesis of increased reporting in Japan. Pink represents over-reported, red represents significantly over-reported $p<0.05$, light blue represents under-reported, blue represents significantly under-reported. ADRs have been sorted by SOC term. For clarification of the SOC abbreviations, see Supplementary Table 1 in the ESM. b Treemap of all reported ADRs over-reported in Japan for irinotecan. Irinotecan disproportionately over-reported PTs in Japan vs RoW, shown in blocks by MedDRA SOC. Block size reflects the frequency of reports in Japan, and colour reflects $\mathrm{IC}_{\Delta}$. Statistical significance of $I C_{\Delta}$ over-reporting: $* p<0.05$, $* * p<0.01, * * * p<0.001$.

ADRs adverse drug reactions, $H M P$ harmonic mean $p$-value, $P T s$ preferred terms, $R o W$ rest of the world, SOC System Organ Class

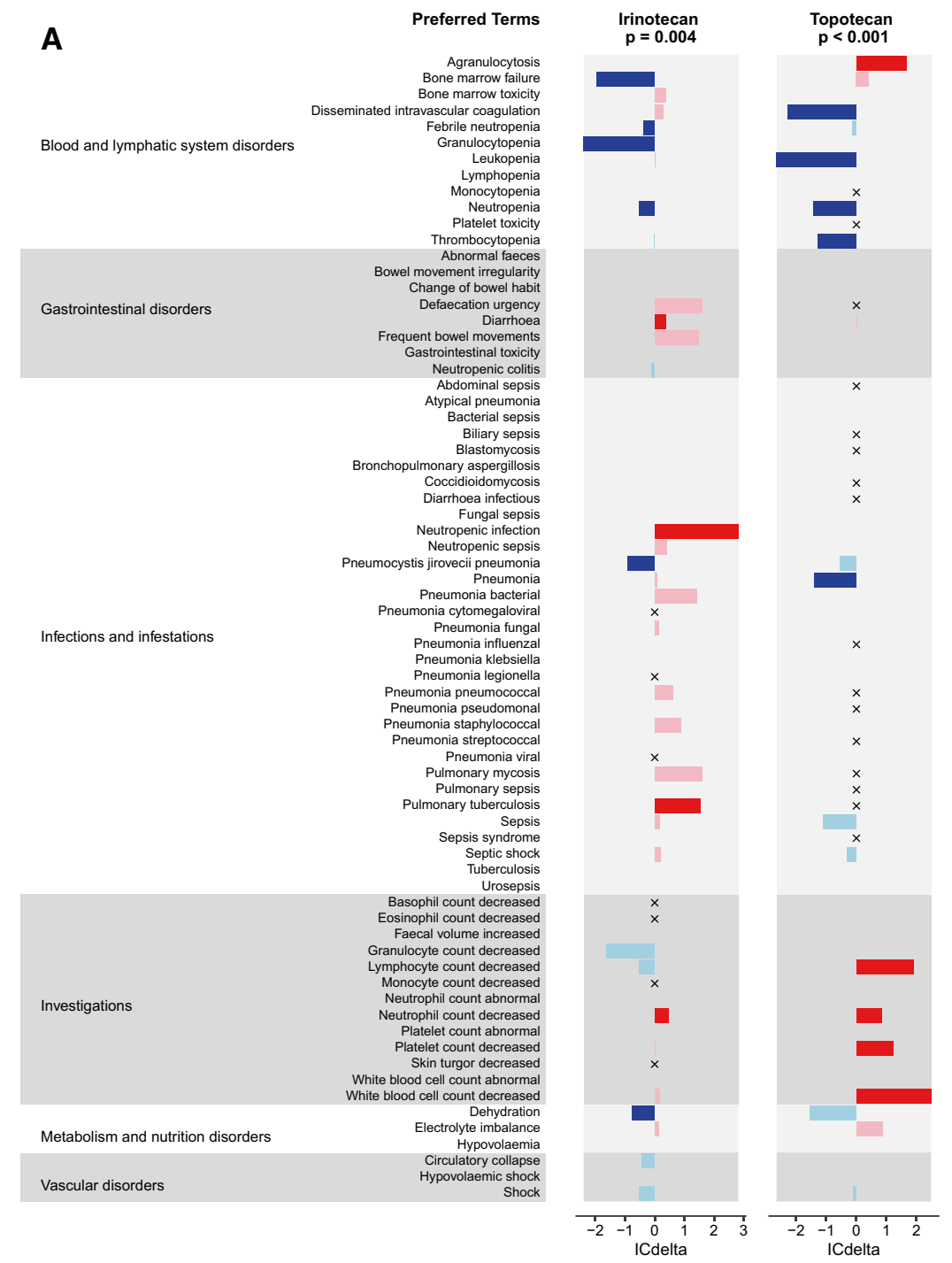

b

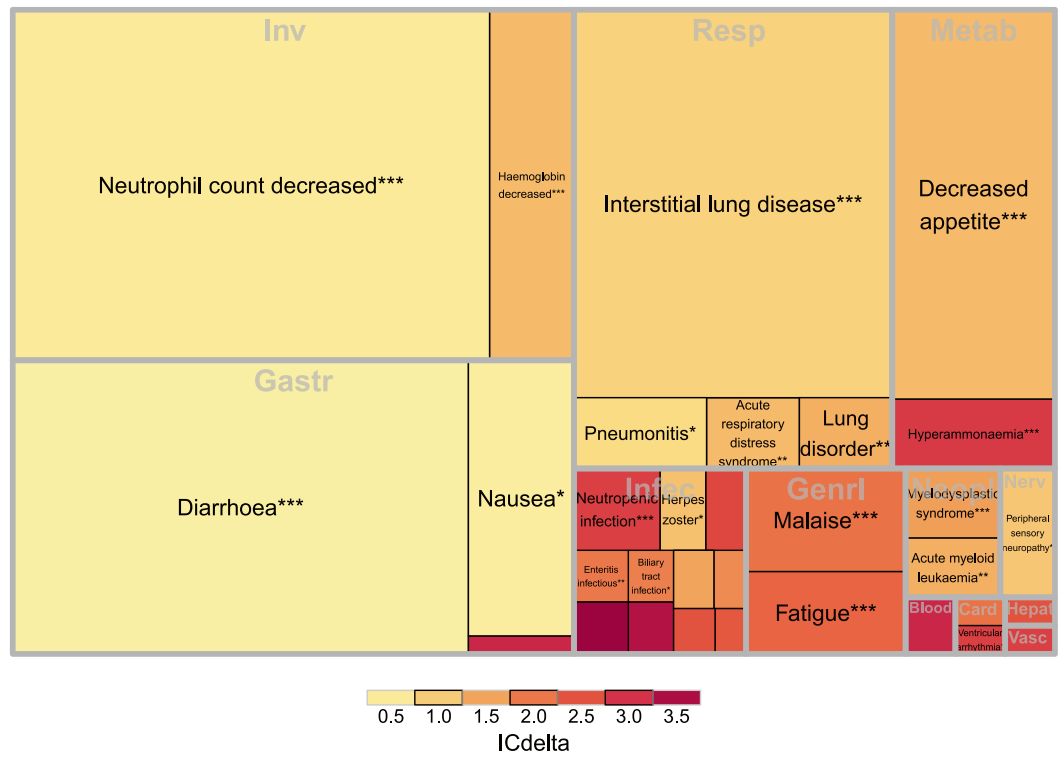


to an $\mathrm{N}$-demethylated metabolite with $60 \%$ of topotecan excreted to urine in unchanged drug.

Voriconazole is a broad-spectrum antifungal agent that belongs to the drug class of triazole antifungals. The triazoles are known to be associated with liver dysfunction. We preselected PTs related to liver disorder, neurological disorder and vision disorder (Supplementary Table 2, see ESM), which are known to be associated with blood concentration of voriconazole [16]. Fluconazole and fosfluconazole were chosen as comparator triazoles as they are not metabolized by CYP2C19 and fosfluconazole is hydrolysed to fluconazole and $77 \%$ of the fluconazole is renally excreted as unchanged drug.

Escitalopram is an antidepressant belonging to a class of drugs known as selective serotonin reuptake inhibitors (SSRIs). It is known to induce dose-dependent QT prolongation [17]. We preselected QT-prolongation-related PTs and overdose-related PTs (Supplementary Table 2, see ESM). The comparator sertraline, an SSRI, is metabolized by CYP2C19, CYP2C9, CYP2B6 and CYP3A4, of which only CYP2C19 has functional differences between populations [18]. Accordingly, we set the other comparator to be milnacipran, a serotonin and norepinephrine reuptake inhibitor (SNRI), which is not metabolized by CYP enzyme, and the Japan label states that $85 \%$ of the drug and its metabolites are renally excreted.

Clopidogrel is a prodrug, one of whose metabolites is an inhibitor of platelet aggregation. The Clinical Pharmacogenetics Implementation Consortium (CIPC) guideline for clopidogrel describes a diminished antiplatelet effect in patients with two loss-of-function alleles of the CYP2C19 gene [19]. We didn't choose cardiovascular events, such as myocardial infarction, stroke or stent thrombosis, as primary biomarkers since lack of effect is not subject to reporting as an ICSR in Japan. Instead, we chose haemorrhage and related terms, predicting them to be under-reported, as surrogate markers (Supplementary Table 2, see ESM). Prasugrel was chosen as a comparator drug, as it is a prodrug and is metabolized by carboxylesterase and CYP3A and CYP2B6 to its active form.

For each of the drugs we provide additional detail and follow-up based on VigiBase data, including dose patterns, observed-to-expected ratios of comparable drugs and fatality rates. As special interest PTs for escitalopram, we chose the QT-prolonged related PTs according to the International Council for Harmonisation (ICH) E-14 Guideline on The Clinical Evaluation of QT/QTc Interval Prolongation and Proarrhythmic Potential for Nonantiarrhythmic Drugs [20].

\section{Results}

The total number of reports included in the analyses from the Japan subgroup was 386,871 , which had been received into VigiBase between December 1972 and November 2019. The total number of reports from the RoW subgroup was $6,453,554$, which had been received into VigiBase between April 1960 and January 2020. Table 1 also includes the number of reports for each of the analysed drugs from both Japan and RoW subgroups.

\subsection{Irinotecan}

The HMPs indicate that the $\mathrm{IC}_{\Delta}$ of the selected PTs were collectively significantly over-reported for both irinotecan (HMP 0.38; $p=0.0004$ ) and topotecan (HMP 0.36; $p<0.001$ ), supporting the hypothesis of increased reporting in Japan. For irinotecan, in addition to PT indicative of neutropenia, multiple PTs within the Infections and Infestations SOC were disproportionately more common in Japan than RoW, suggesting an increased severity of myelosuppression. In contrast, topotecan revealed an underreporting of PT associated with infections, and the reporting of diarrhoea was not observed (Fig. 1a, b).

The dosage distribution of irinotecan was lower in Japan compared with RoW (Fig. 2), as was the median daily dose ( $210 \mathrm{mg}$ compared with $240 \mathrm{mg}$, Table 2), corroborating the fact that the approved dosage is lower in Japan than in RoW.

\subsection{Voriconazole}

The HMP for voriconazole showed collectively significant over-reporting (HMP $0.1 ; p<0.001$ ) not seen with

Table 1 The number of reports included in the analyses for each of the selected drugs (in bold) and their comparators by subgroup (Japan and the rest of the world [RoW])

\begin{tabular}{lll}
\hline & Japan & RoW \\
\hline Irinotecan & 2342 & 14,734 \\
Topotecan & 262 & 3028 \\
Voriconazole & 1073 & 7195 \\
Fluconazole & 202 & 6442 \\
Fosfluconazole & 128 & 4 \\
Escitalopram & 538 & 14,428 \\
Milnacipran & 95 & 1081 \\
Sertraline & 731 & 22,067 \\
Clopidogrel & 3437 & 35,489 \\
Prasugrel & 764 & 5200 \\
Reports in entire subset & 386,871 & $6,453,554$ \\
\hline
\end{tabular}

The table also displays the total number of reports from each subgroup included in VigiBase 
fluconazole (HMP 0.84; $p=0.420$ ) or fosfluconazole (HMP $0.095 ; p=0.260$ ), although the latter should not be overinterpreted being based on only four existing PTs. In accordance with the hypothesis, visual and neurological PTs were over-reported with voriconazole; however, the hepatotoxicity-related PTs were under-reported. Furthermore, PTs for 'drug level increased', 'drug ineffective' and 'liver function test abnormal' showed significant over-reporting in Japan (Fig. 3a, b).

The median daily dose of voriconazole was identical in Japan compared with RoW (400 mg, Table 2), but the dosage distributions (Fig. 2) reveal that lower doses are given more commonly in Japan compared with RoW.

\subsection{Escitalopram}

All three drugs revealed significant over-reporting of hypothesized PTs in Japan compared with the RoW (escitalopram: HMP 0.28, $p<0.001$; milnacipran: HMP 0.42, $p=0.004$; sertraline: HMP 0.22, $p<0.001$ ). Sertraline had a larger number of significantly over-reported hypothesized PTs compared with escitalopram, while the treemap for escitalopram revealed an over-reporting for a number
Table 2 Median daily doses for the selected drugs (in bold) and comparator drugs in reports from Japan and the rest of the world (RoW)

of additional PTs, including mania, intentional self-injury, completed suicide and suicidal attempt (Fig. 4a, b).
Fig. 2 Dose distributions for selected drugs and their comparators, from Japanese reports and reports from the rest of the world (RoW). Dosages in $\mathrm{mg} /$ day on the $x$-axis, and probablity density on the $y$-axis
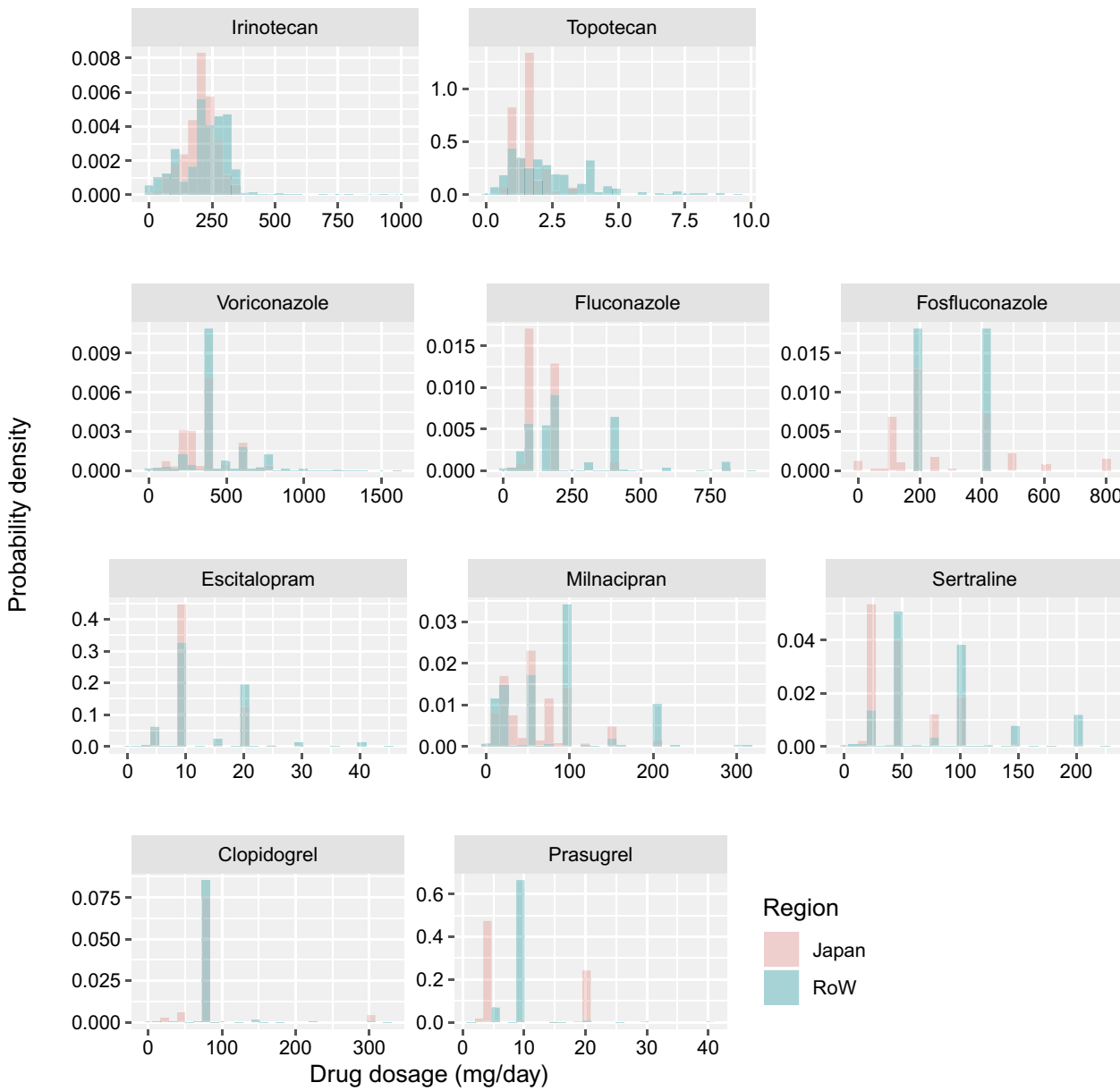
A

Preferred Terms

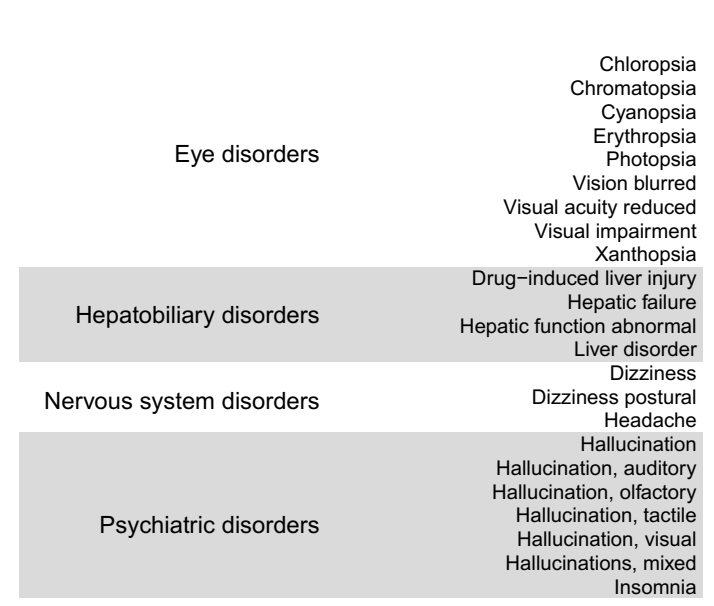

B
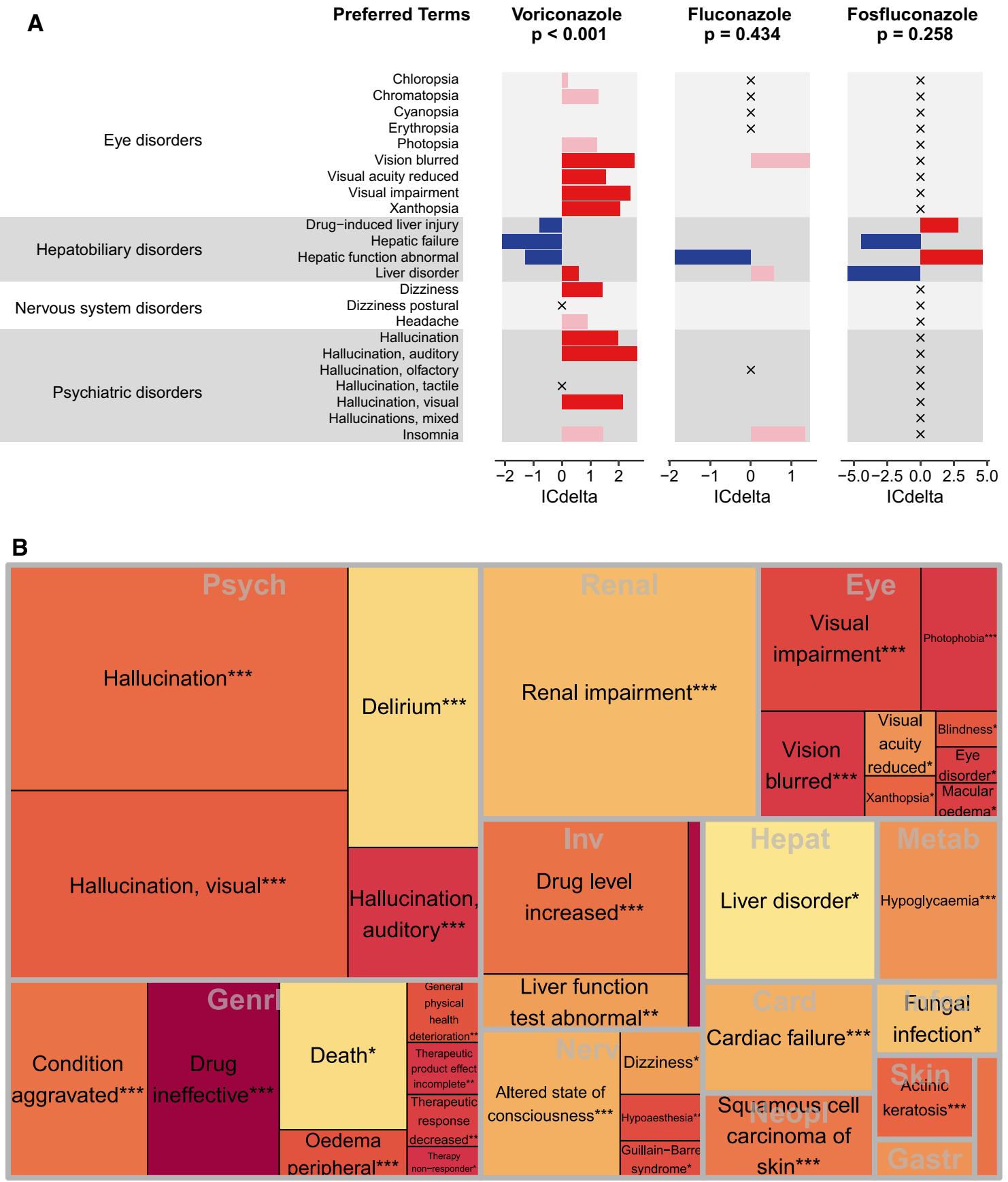

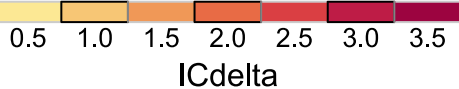

Fig. 3 Voriconazole. a HMP analysis of pre-specified PTs for voriconazole, fluconazole and fosfluconazole. Drug-specific ADRs were selected prior to the study as suspected for over-reporting in Japan compared with RoW if the hypothesis of increased risk to Japanese were true. An HMP $<0.05$ indicates that the $\mathrm{IC}_{\Delta} s$ of the preselected PTs are collectively significantly over-reported, supporting the hypothesis of increased reporting in Japan. Pink represents overreported, red represents significantly over-reported $p<0.05$, light blue represents under-reported, blue represents significantly underreported. ADRs have been sorted by SOC term. Only four of the 


\subsubsection{Risk Summary of Escitalopram Special Interest Preferred Terms}

Further comparative analyses of PTs related to QT prolongation revealed that each of the PTs of electrocardiogram QT prolonged, long QT syndrome, syncope, torsade de points and ventricular fibrillation were estimated to be overreported compared with other drugs within Japan (IC > 0, all are statistically significant with a $5 \%$ significance level except for syncope and torsade de pointes, for which the $95 \%$ credibility intervals overlap slightly with 0 ); furthermore, although the reporting of electrocardiogram QT prolonged, long QT syndrome and syncope for escitalopram exceeded those of both milnacipran and sertraline, none of these differences in IC were statistically significant. Finally, there was no evidence of an increased proportion of fatal reports of electrocardiogram QT prolonged for escitalopram compared with sertraline or milnacipran (Fig. 5a, b).

The daily dosages registered with escitalopram revealed identical median doses in Japan and RoW (10 mg, Table 2), but dose distributions (Fig. 2) suggest considerably higher doses are more often registered in RoW, in line with the lower dosage recommendation in the Japanese label.

\subsection{Clopidogrel}

We predicted that decreased bleeding would be detected preferentially in clopidogrel. The decreased risk of bleeding was evident from under-reporting of PTs in the haemorrhagic standardized MedDRA query (SMQ) (specifically gastrointestinal and neurological). Clopidogrel had a larger number of significantly under-reported PTs than prasugrel. However, both drugs had collectively significant underreporting of hypothesized PTs in Japan versus the RoW (for clopidogrel, HMP $=0.26, p<0.001$; for prasugrel, HMP = $0.47, p=0.020$ ) (Fig. 6a, b).

Lower median daily dosages of prasugrel are reported from Japan compared with RoW (3.75 mg compared with $10 \mathrm{mg}$, Table 2), although dose distribution shows that higher dosages in Japan are also being used (Fig. 2).

\section{Discussion}

The results of our investigation suggest that subgroup disproportionality analyses within a global database of ICSRs can detect phenotypes of harm secondary to pharmacogenomic risk variants common in Japan. Predicted ADRs for irinotecan, voriconazole, escitalopram and clopidogrel were observed with higher (or lower) levels of reporting in Japan within a global database of ICSRs. Furthermore, more extensive analyses of ICSRs of adverse drug events could be used to explore regulatory concerns specific to Japan during post-licensure use and risk mitigation tools as prescribed by the Pharmaceuticals and Medical Devices Agency (PMDA), such as dosage recommendations and therapeutic drug monitoring.

\subsection{Irinotecan}

Our analysis revealed that PTs for irinotecan-related toxicities were significantly over-reported in spite of the lower dosage distribution in Japan compared with RoW, in sharp contrast to topotecan, for which there was underreporting of the infections PT and no observable reporting of diarrhoea. The overall pattern in over-reported PTs is concordant with other literature that demonstrate that the positive association of the UGT1A $1 * 6$ allele is more common for neutropenia $[21,22]$ than for diarrhoea. UGT1A1 $* 6$ has allele frequencies of $15 \%$ among Japanese population [23], and there is evidence that UGT $1 \mathrm{~A} 1 * 6$ rather than UGT $1 \mathrm{~A} 1 * 28$ contributes to irinotecan-related toxicity in Japanese [24]. Our results on dosage distribution are supported by the fact that the approved dosage for irinotecan in the US ranges from $125 \mathrm{mg} / \mathrm{m}^{2} /$ week to $350 \mathrm{mg} / \mathrm{m}^{2}$ triweekly [25], while the maximum approved dose is $180 \mathrm{mg} / \mathrm{m}^{2}$ biweekly in Japan [26]. Our analysis points to the importance of the UGT1A $1 * 6$ vulnerability of Japanese to irinotecan-induced toxicity.

The spontaneous reporting of these ADRs in Japan confirms the major trend found in the regulatory all-case survey of irinotecan from April 1995 to January 2000 [15]. It was conducted for 13,935 patients treated with irinotecan, and it revealed the major grade 3-4 ADRs to be leukopenia (34.8\%), thrombocytopenia (12.4\%) and diarrhoea (10.1\%). Since UGT1A1 pharmacogenetic testing was introduced in 2008 in Japan, there is the possibility that the risk has been better managed. However, reporting of irinotecan-related toxicities continues, which could be explained by the paucity of evidence and guidance on genotype-directed dosing.

Pharmacogenetic testing for irinotecan is recommended in guidelines or labels from the US Food and Drug Administration, European Medicines Agency and Japan's PMDA [4]. Yet, while the UGT1A1 testing is covered by the Japanese national insurance, a survey revealed that pharmacogenomic UGT1A1 testing was rarely covered by the major health insurers in the US [27]. Although the difference in health insurance coverage between Japan and the US could theoretically influence our results, our analysis still detected the over-reporting in Japan, suggesting the vulnerability of Japanese. 
Fig. 4 Escitalopram. a HMP analysis of pre-specified PTs for escitalopram, milnacipran and sertraline. Drug-specific ADRs were selected prior to the study as suspected for over-reporting in Japan compared with RoW if the hypothesis of increased risk to Japanese were true. An HMP $<0.05$ indicates that the $\mathrm{IC}_{\Delta} \mathrm{s}$ of the preselected PTs are collectively significantly over-reported, supporting the hypothesis of increased reporting in Japan. Pink represents over-reported, red represents significantly over-reported $p<0.05$, light blue represents under-reported, blue represents significantly under-reported. ADRs have been sorted by SOC term. All three drugs have significant over-reporting of hypothesized PTs in Japan compared with the RoW. Sertraline has a larger number of significantly over-reported PTs (7 vs 3). b Treemap of all reported ADRs over-reported in Japan for escitalopram. Escitalopram disproportionally over-reported PTs in Japan vs RoW, shown in blocks by MedDRA SOC. Block size reflects the frequency of reports in Japan, and colour reflects $\mathrm{IC}_{\Delta}$. Statistical significance of $\mathrm{IC}_{\Delta}$ over-reporting: $* p<0.05$, $* * p<0.01$, $* * * p<0.001$. ADRs adverse drug reactions, $H M P$ harmonic mean $p$ value, $P T s$ preferred terms, RoW rest of the world, SOC System Organ Class

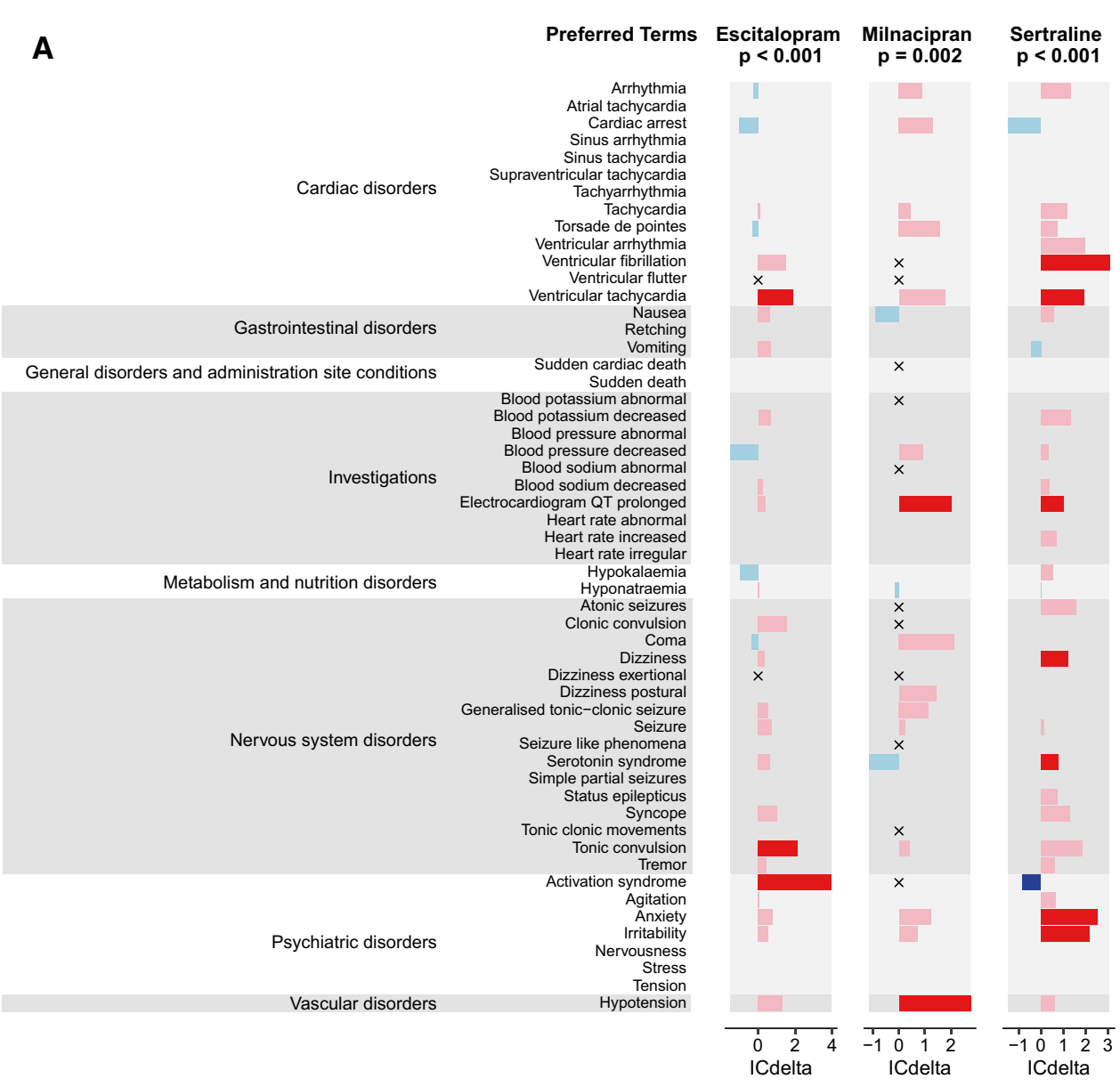

B

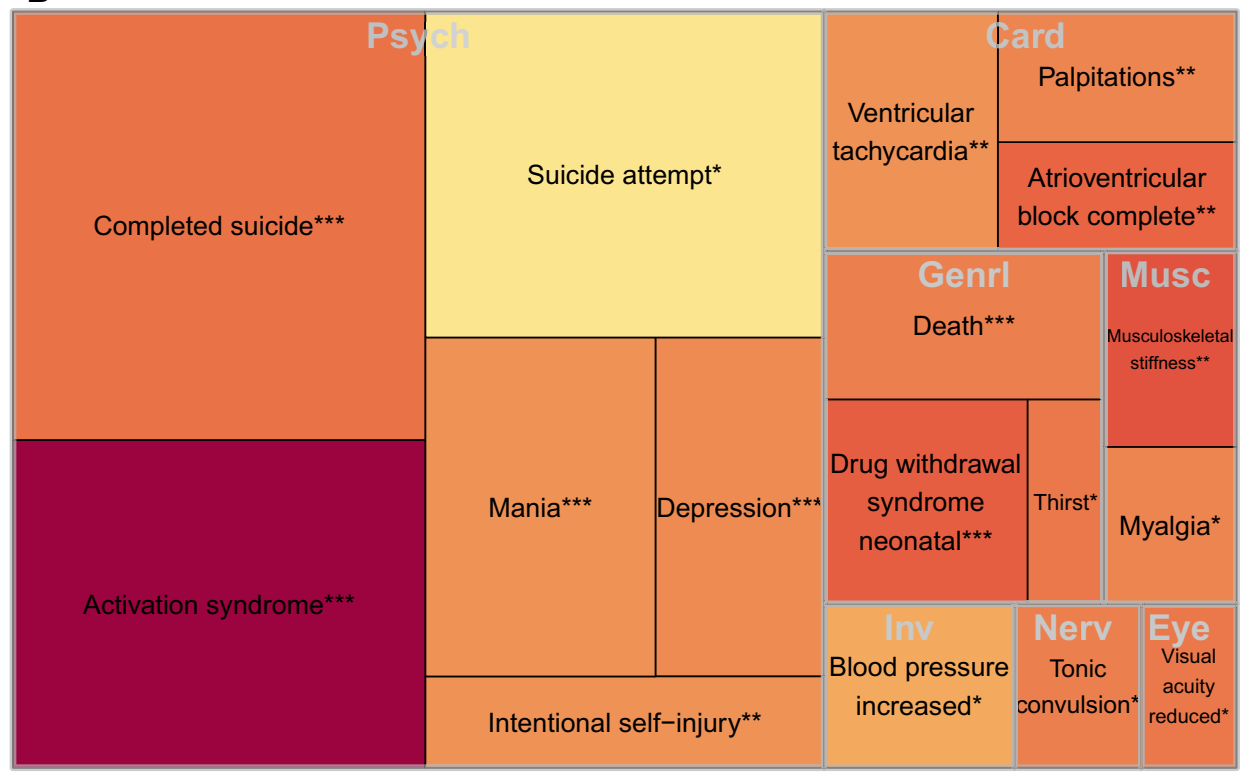



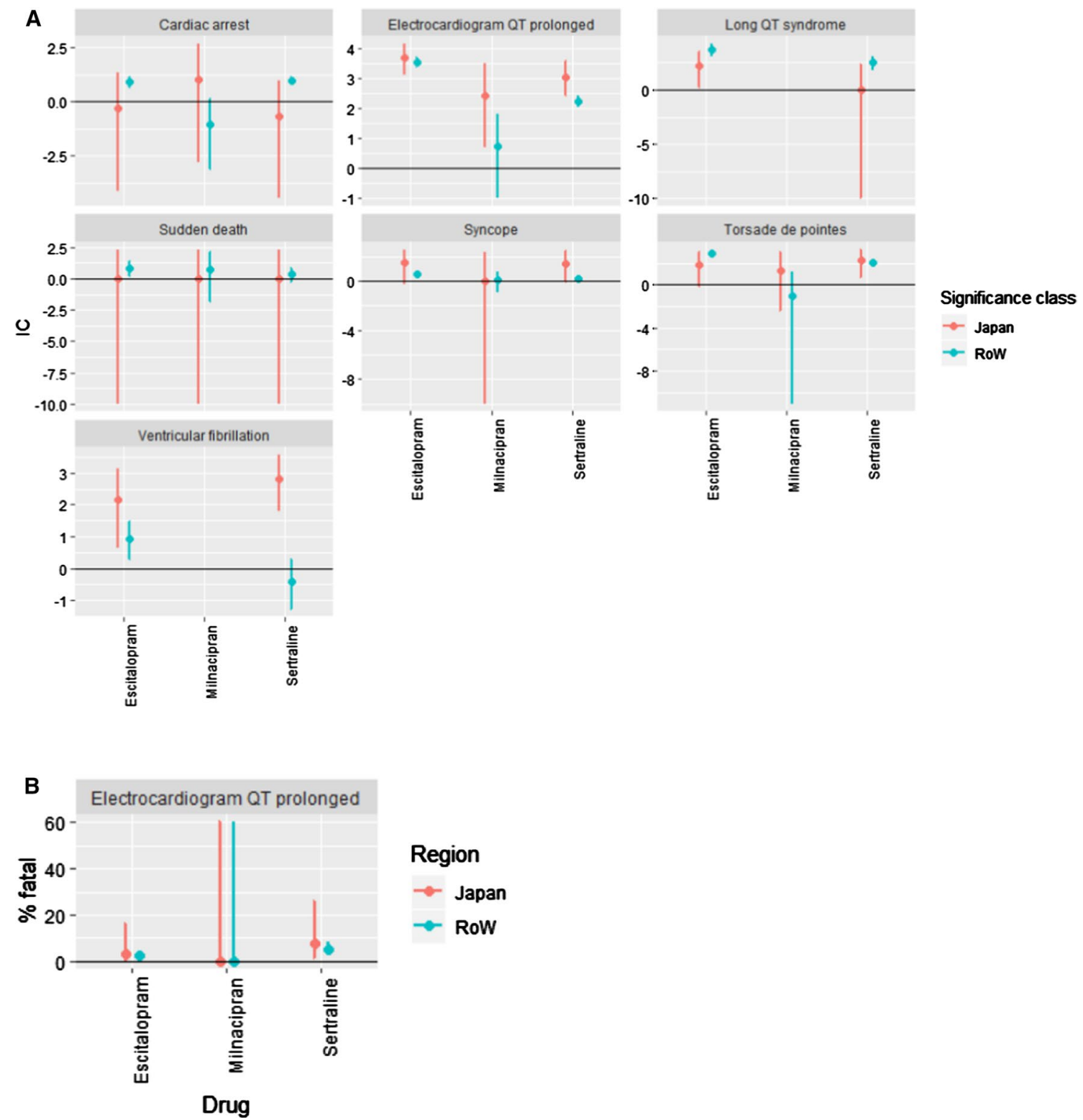

Fig. 5 Risk summary of escitalopram special-interest PTs. a Disproportionality investigation of PTs related to QT prolongation with escitalopram and comparator drugs. IC shows a robust observed-toexpected rate of a PT on a selected drug, comparing the number of observed reports to that expected given the reporting rate of that PT on all drugs (within Japan, or within RoW, respectively). Error bars reflect $95 \%$ credibility intervals of IC. 'Electrocardiogram QT prolonged' was shortened to 'QT prolonged'. b Proportion of 'Electro-

Fig. 6 Clopidogrel. a HMP analysis of pre-specified PTs for clopidogrel and prasugrel. Drug-specific ADRs were selected prior to the study as suspected for over-reporting in Japan compared with RoW if the hypothesis of increased risk to Japanese were true. An HMP < 0.05 indicates that the $\mathrm{IC}_{\Delta} \mathrm{s}$ of the preselected PTs are collectively significantly over-reported, supporting the hypothesis of increased reporting in Japan. Pink represents over-reported, red represents significantly over-reported $p<0.05$, light blue represents underreported, blue represents significantly under-reported. ADRs have been sorted by SOC term. Both drugs have significant under-report- cardiogram QT prolonged' fatal case reports on escitalopram, milnacipran and sertraline, with $95 \%$ confidence intervals (vertical lines). 'Electrocardiogram QT prolonged' is the only PT on our special interest list for escitalopram that has a fatal report. Statistically, there is no sign of escitalopram reports having a higher rate of fatal cases within Japan than the comparator drugs, as the confidence intervals in the upper panel are all overlapping. PTs preferred terms

ing of hypothesized PTs in Japan versus the RoW, but we see many more significantly under-reported PTs with clopidogrel, in particular within the 'Gastrointestinal disorders' and 'Nervous system disorders' SOCs. b Treemap of all reported ADRs over-reported in Japan for clopidogrel. Clopidogrel disproportionally over-reported PTs in Japan vs RoW, shown in blocks by MedDRA SOC. Block size reflects the frequency of reports in Japan, and colour reflects $\mathrm{IC}_{\Delta}$. Statistical significance of $\mathrm{IC}_{\Delta}$ over-reporting: $* p<0.05, * * p<0.01$, *** $p<0.001$. $A D R s$ adverse drug reactions, HMP harmonic mean $p$ value, $P T s$ preferred terms, RoW rest of the world, SOC System Organ Class 
A

Blood and lymphatic system disorders

Cardiac disorders

Congenital, familial and genetic disorders Ear and labyrinth disorders

Endocrine disorders

Eye disorders

Gastrointestinal disorders

Blood loss anaemia (avcular coagulatio Haemorrhagic diathesis Immune thrombocytopenic purpura Increased tendency to bruise Splenic haematoma Splenic haemorrhage Spontaneous haematoma Spontaneous haemorrhage Thrombocytopenic purpura Thrombotic thrombocytopenic purpura Haemorrhage coronary artery Myocardial haemorrhage Myocardial rupture Ventricle rupture Cerebral arteriovenous malformation haemorrhagic Haemorrhagic arteriovenous malformation Haematotympanum Adrenal haemorrhage Haemorrhagic thyroid cyst Pituitary apoplexy Pituitary haemorrhage Thyroid haemorrhage Choroidal haemorrhage Ciliary body haemorrhage

Corneal bleeding Eye haemorrhage Eyelid haematoma ntraocular haematoma Iris haemorrhage Lacrimal haemorrhage Ocular retrobulbar haemorrhage Retinal haemorrhage Scleral haemorrhage Vitreous haemorrhage Abdominal wall haematoma Anal haemorrhage Anal haemorrhage Anal ulcer haemorrhage Chronic gastrointestinal bleeding Colonic haematoma Diarrhoea haemorrhagic Diverticulum intestinal haemorrhagic

Duodenal ulcer haemorrhage

Duodenitis haemorrhagic

Enterocolitis haemorrhagic Gastric haemorrhage Gastric ulcer haemorrhage Gastric ulcer perforation Gastritis haemorrhage Gastritis haemorrhagic Gastroduodenal haemorrhage Gastestinal polyp haemorrhage Gastrointestinal ulcer haemorrhage Gastrointestinal vascular malformation haemorrhagic Gingival bleeding Haematemesis Haematochezia Haemorrhagic ascites Haemorrhagic erosive gastritis emorrhagic necrotic pancreatitis

Intidal haemorrhag Intestinal haematoma Intestinal haemorrhage Intestinal varices haemorrhage Intra-abdominal haemorrhage Large intestinal haemorrhage Large intestinal ulcer haemorrhage Lip haematoma
Lip haemorrhage Lip haemorrhage
Lower gastrointestinal haemorrhage Mallory-Weiss syndrome Melaena
Mesenteric haematoma Mesenteric haematoma
Mouth haemorrhage

Mouth haemorrhage
Nausea

Oesophageal haemorrhage Oesophageal intramural haematoma Oesophageal ulcer haemorrhage Oesophageal varices haemorrhage Pancreatic haemorrhage Pancreatitis haemorrhagic Peptic ulcer haemorrhage Peritoneal haematoma Peritoneal haemorrhage roctitis haemorrhagic Rectal haemorrhage emorrhage

Retching
Retroperitoneal haematoma Small intestinal haemorrhage Small intestinal ulcer haemorrhage Tongue haematoma Tongue haemorrhage Tooth pulp haemorrhag Tooth socket haemorrhage Upper gastrointestinal haemorrhage
Vomiting
Clopidogrel Prasugrel $p<0.001 \quad p=0.020$

-

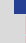

II

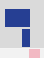

$\times$

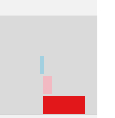

$\square$

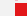

$-$

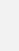

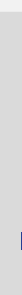

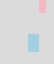

1

$\times$

$\times$
$\times$

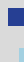

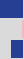

.

|

$\times$
$\times$
$x$
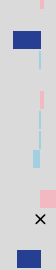
General disorders and administration site conditions

Administration site haemorrhage Application sile haematoma Application site haemorrhage Catheter site haematoma Catheter site haemorrhage Haemorrhagic cyst mplant site bruising mplant site haematoma Implant site haemorrhage Infusion site haematoma Infusion site haemorrhage Injection site bruising Injection sile haematoma Injection ste haemorrhage Medical device site haemorrhage Mucosal haemorrhage Puncture site haemorrhage Ulcer haemorrhage Vessel puncture site bruise Vessel puncture site haematoma Vessel puncture site haemorrhage

Haemorrhagic hepatic cyst Hepatic haematoma Hepatic haemorrhage Diverticulitis intestinal haemorrhagic Haematoma infection

Anastomolic haemorrhage Arteriovenous fistula site haemorrhage Brain contusion Contusion Deep dissecting haematoma
Epidural haemorrhage Extradural haematoma Graft haemorrhage Graft haemorrhage Haematuria traumatic

Incision site Hyphaema Incision site haematoma Kidney contusion Periorbital haematoma Periorbital haemorrhage Perirenal haematoma Injury, poisoning and procedural complications

Post procedural ha Post procedural haematuria Post procedural haemorrhage Procedural haemorrhage Pulmonary contusion Scrotal haematoma Stoma site haemorrhage Subarachnoid haem

Subculangus haematoma Subdural haematoma Tracheal haemorrhage Traumatic haematoma Traumatic haemorrhage Traumatic haemothorax Traumatic intracranial haematoma raumatic intracranial haemorrhage Vascular access site haematoma Vascular access site haemorrhage Vascular pseudoaneurysm ruptured Wound haematom Wound haemorrhage
Blood urine Blood urine present Chest wall haematoma Haemarthrosis Haematoma muscle Muscle haemorrhage Soft tissue haemorrhage

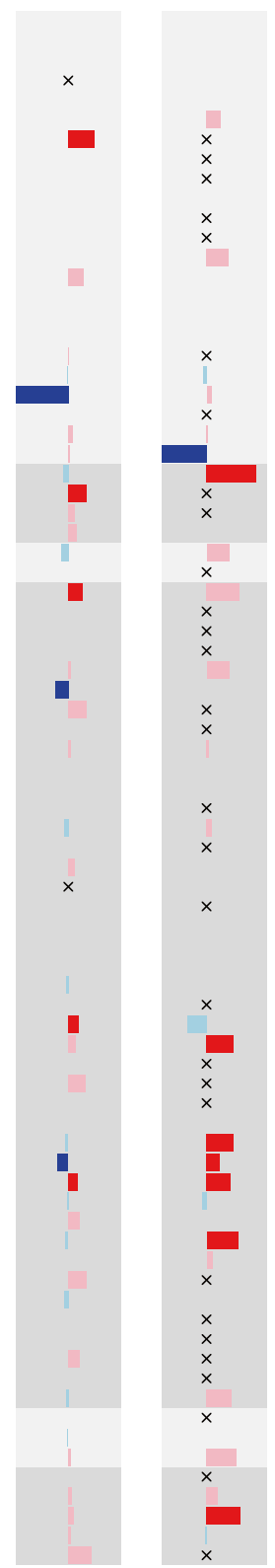

Fig. 6 (continued)

\subsection{Voriconazole}

Our analysis revealed that concentration-dependent events for voriconazole are collectively over-reported while slightly lower dosages are used in Japan compared with RoW, in marked contrast to non-significant over-reporting of comparators' events. More specifically, there was an over-reporting of visual and neurological PTs but also an under-reporting of hepatotoxicity-related PTs in Japan compared with the RoW. Liver disorder, neurological disorder and vision disorder are known to be associated with blood concentration of voriconazole [16, 28]. However, given the complex nature of patients treated with voriconazole, it is acknowledged that additional factors beyond CYP2C19 polymorphism, such as drug interactions and other comorbidities, can affect blood concentration levels. Further support is provided by the inter-patient variability in blood levels among the same CYP2C19 genotypes in clinical studies submitted to preapproval review.

Our observations of an over-reporting of visual and neurological adverse events in Japan are consistent with previously published data. In prelicensure phase III trials, visual ADRs were observed in 52\% of Japanese versus $20.3 \%$ of foreign subjects [6]. Published literature suggests that 


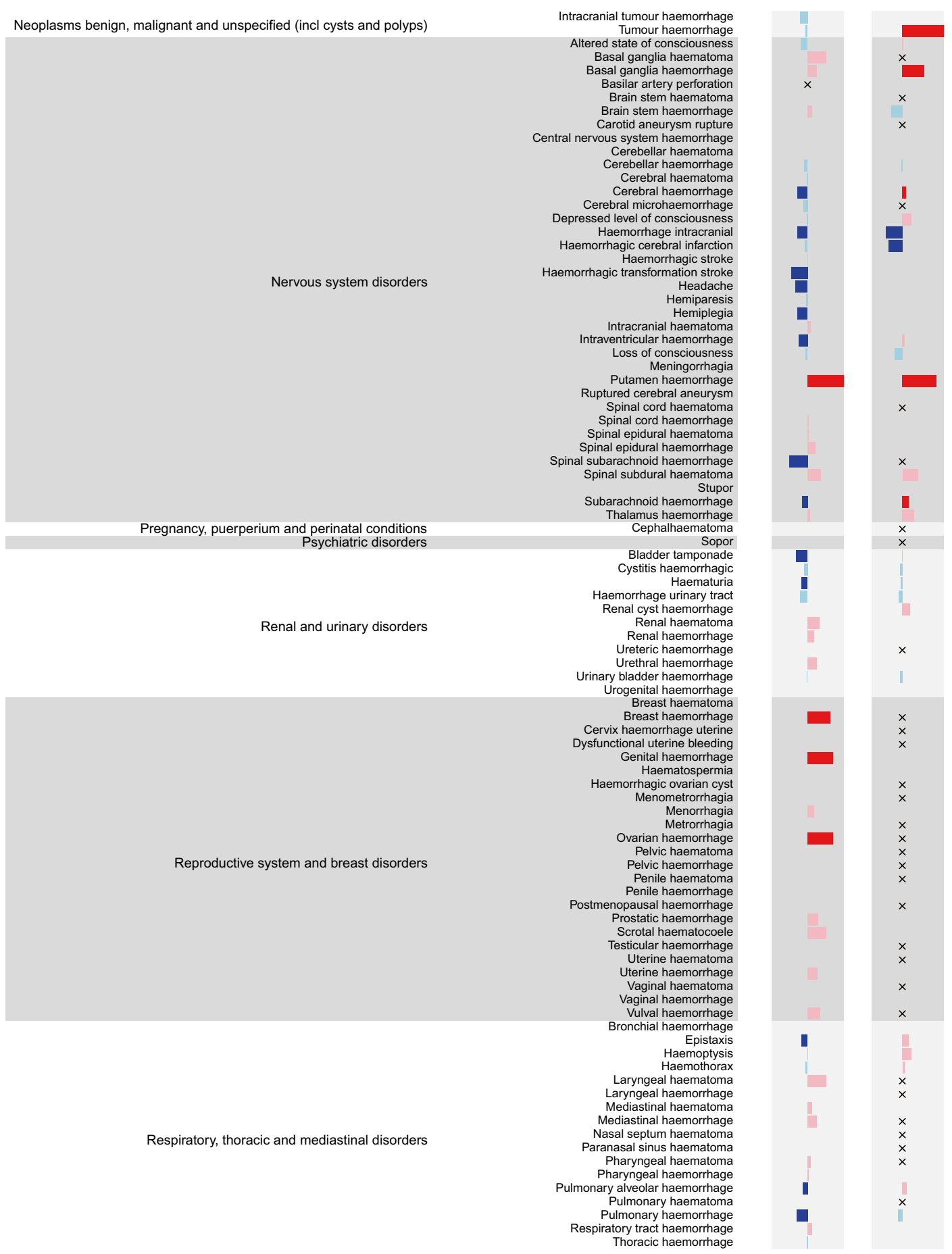

Fig. 6 (continued)

voriconazole trough concentrations may influence a patient's risk of developing neurological toxicities [16], and a case series reported their occurrence with a trough level $<5 \mu \mathrm{g} /$ $\mathrm{mL}$, the threshold for liver disease [29].

The under-reporting of hepatotoxicity-related PTs could be explained by pharmacovigilance activities in Japan. The higher rate of visual and hepatotoxicity-related ADRs in
Japanese observed in prelicensure trials led to a warning for hepatic ADRs in the product label. Additionally, given the wide variation of blood levels among subjects in prelicensure trials and the high prevalence of CYP2C19 poor metabolizers in Japan, the product label includes the recommendation to use both liver function testing and therapeutic drug monitoring (TDM) to guide decisions on dosing. The 


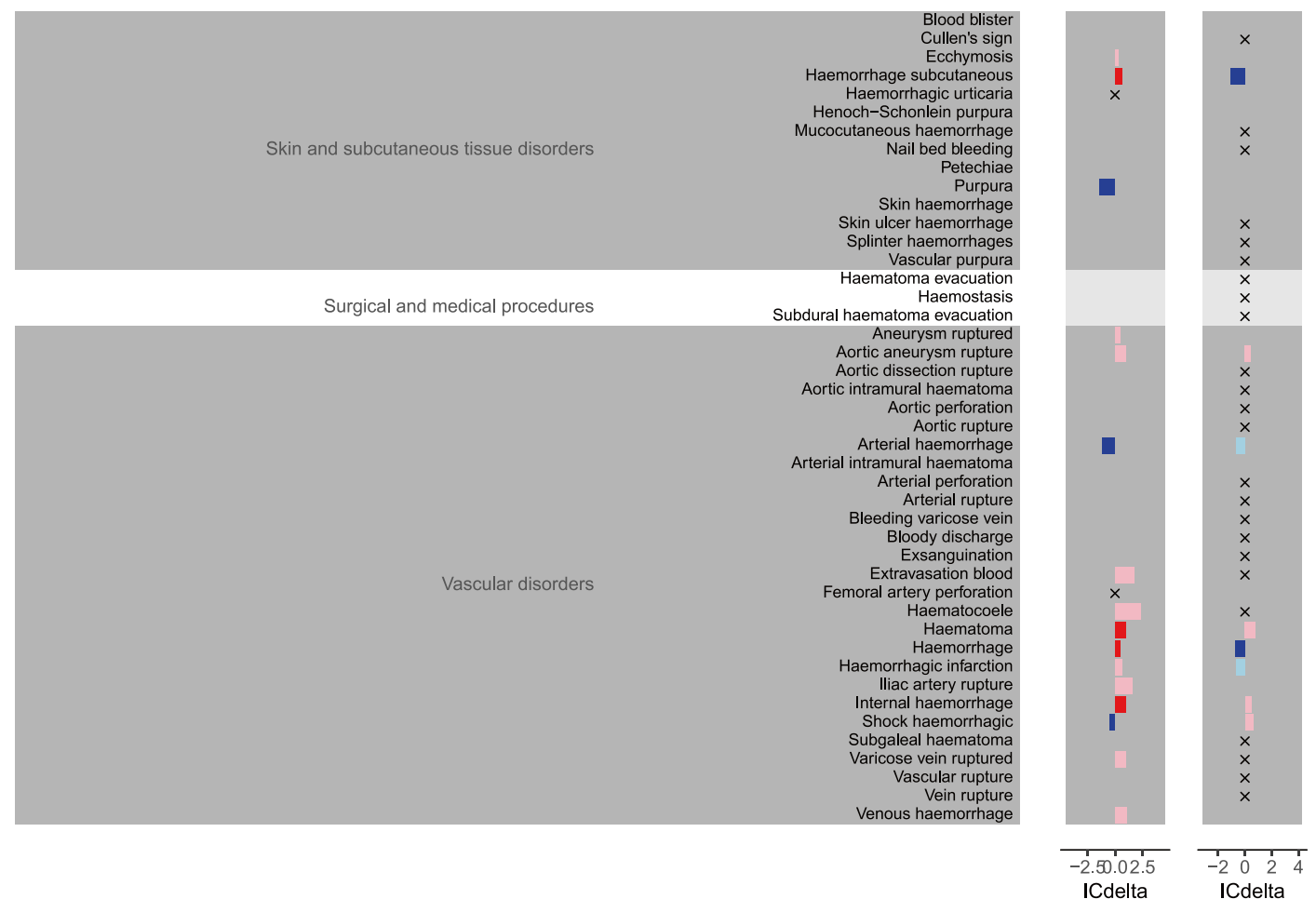

B

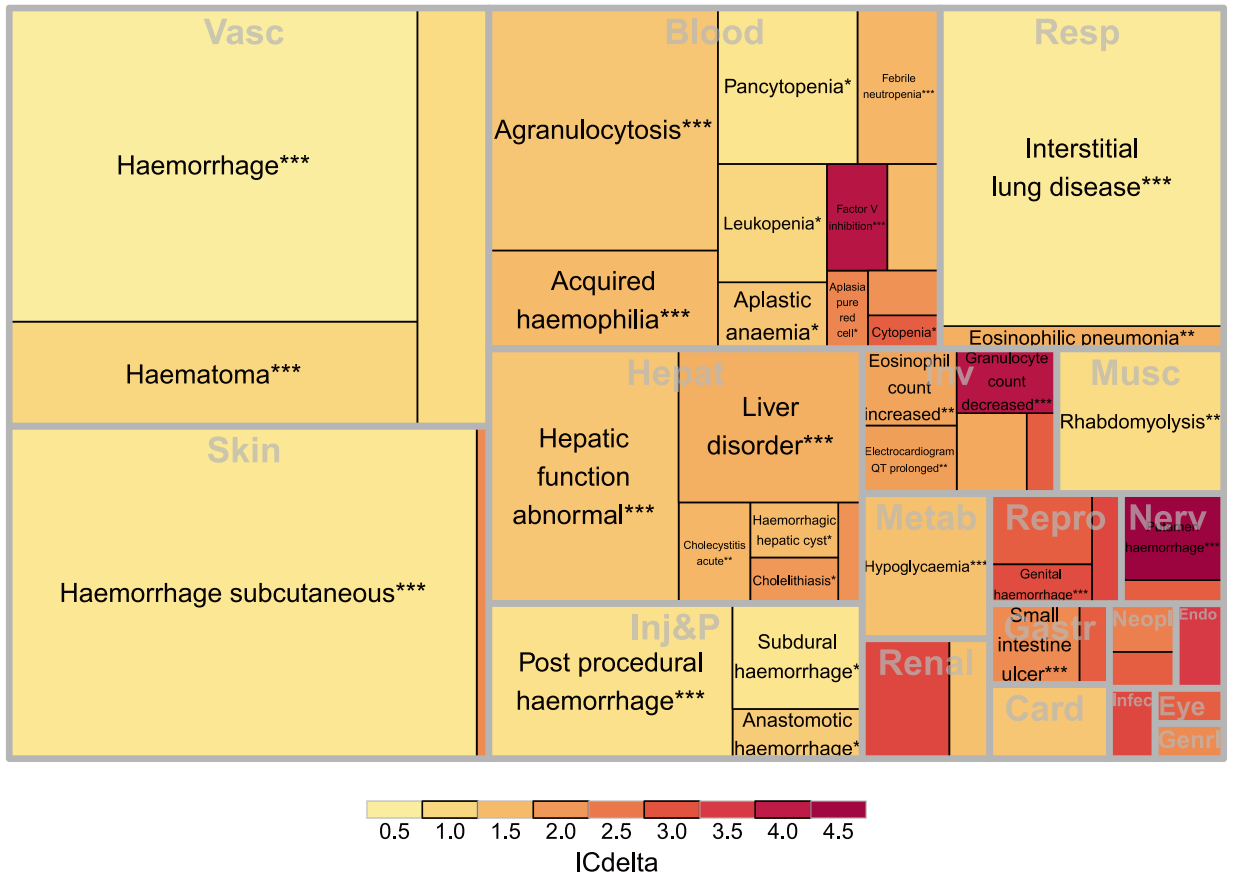

Fig. 6 (continued)

TDM guideline in Japan specifies the use of TDM in case where there is either a lack of clinical effects or the presence of toxicities [30]. The target trough concentration is $\geqq 1-2 \mu \mathrm{g} / \mathrm{mL}$ with a caution for liver disorder if the concentration is $\geqq 4-5 \mu \mathrm{g} / \mathrm{mL}$ [28]. These safety measures are reflected in spontaneous reporting, as our results reveal the PTs for 'drug level increased', 'drug ineffective' and 'liver function test abnormal' are significantly over-reported in Japan (combined with the under-reporting of hepatic toxicity) compared with the RoW. 


\subsection{Escitalopram}

Our analysis did not find any evidence of increased reporting of the concentration-dependent ADR of QT prolongated for escitalopram. Although there was evidence of greater disproportionality of reporting for the specific PTs of electrocardiogram QT prolonged, long QT syndrome and syncope compared with both milnacipran and sertraline within Japan, none of these differences were statistically significant. Furthermore, there was no evidence of an increased fatal proportion of the ADR QT prolonged in escitalopram. Also, fewer PTs from the Cardiac disorders SOC were overreported for escitalopram than sertraline. These results suggest the cardiovascular effects inherent to SSRIs are overreported in Japan without increased risk of death related to QT prolongation, and the cardiac risks of escitalopram appear to be within the range of those of sertraline. The overreporting of some events observed in both escitalopram and its comparator can be explained by the partial CYP2C19dependent metabolism of sertraline. QTc prolongation may also be explained by the coexistence of other factors, such as hypokalaemia and hypomagnesaemia [20].

It is reassuring that the safety profile regarding QT-related events of escitalopram is similar to that of sertraline in Japanese. Regulatory actions at the time of approval included dosing guidance in the labelling ("It is preferable to use the upper limit of 10mg for CYP2C19 poor metabolizer, since the blood level of escitalopram could increase and lead to ADRs such as QT-prolongation.”) and inclusion of QT prolongation within the risk management plan for escitalopram [7]. These measures may lead to clinical use of escitalopram at a lower dosage, so that CYP2C19 poor metabolizers do not develop dose-related QT prolongation.

\subsection{Clopidogrel}

Our results revealed that both clopidogrel and prasugrel had collectively significant under-reporting of bleeding PTs in Japan versus the RoW ( $p<0.001$ for clopidogrel, $p=0.020$ for prasugrel). Our prediction of a relatively decreased risk of bleeding for clopidogrel over prasugrel was supported by the under-reporting of PTs in the haemorrhagic SMQ, seen particularly in gastrointestinal and neurological bleeding, which are the most clinically important sites of bleeding associated with its use. We see under-reporting of prasugrel for the same bleeding PTs, in line with the lower median daily dosages in Japan compared with RoW (3.75 mg compared with $10 \mathrm{mg}$, Table 2). East-Asian patients have different risk profiles for both thrombophilia and bleeding compared with White patients, and a different 'therapeutic window' of on-treatment platelet reactivity might be appropriate in East-Asian patients [31]. The approved dosage of prasugrel in Japan is one third of the approved dosage in the
US (60 mg loading dose with a $10 \mathrm{mg}$ maintenance dose in the US compared to, a 20-mg loading dose and a $3.75-\mathrm{mg}$ maintenance dose in Japan [32, 33]. Of note, a meta-analysis has suggested that the outcome of major adverse cardiovascular events, in contrast to bleeding, is associated with poor metabolization by CYP2C19, indicating that further analysis using thrombotic ADRs may be warranted [34].

In only one of the examples was there a clear difference in HMP between the selected drug and its comparator drug(s). The observed over-reporting of prespecified events in some comparator drugs could be explained by the fact that some of the pre-specified events could be the result of drug class effects, such as myelosuppression (topotecan), which is common in topoisomerase inhibitors, and cardiovascular effects (milnacipran and sertraline), which are common in SSRI and SNRI. Alternatively, the largest difference in HMP was seen between voriconazole and fluconazole/fosfluconazole; indications for the use of these drugs and thus underlying patient populations may differ more markedly in this case than in the other comparisons.

\subsection{Adding New Dimensions}

Important challenges in the review of applications for marketing approval of pharmaceuticals are the prediction of drug usage in the real world and appropriate risk minimization, as post-approval use includes populations never examined in the pre-approval clinical trials. Our study has revealed that analyses of regional data in a global context can inform the effectiveness of local risk minimization measures.

\subsection{Limitations}

Limitations to our study largely reflect the nature of ICSR data. Neither estimations nor comparisons of the incidence of ADRs are possible given the absence of denominator data on number of users. Lower median dosages of all drugs (study drugs and comparators) were used in Japan compared with the rest of the world, limiting our ability to draw strong conclusions on the impact of the risk management recommendation of lower dosing. However, information on dosing in reports of ADRs is often missing. An internal estimate reveals that only $8 \%$ of the VigiBase reports provide sufficient information for derivation of a total daily dose; therefore, dose distributions were derived from only the small total number of reports for each drug. Analyses within such databases are largely limited to observations of reporting patterns for ADRs, which can be influenced by multiple factors, both drug-level and patient-level. Finally, the occurrence of an ADR is a manifestation of a phenotype that can suggest, but not predict, an underlying genotype. 


\section{Conclusion}

Pharmaco-ethnic vulnerabilities caused by pharmacogenomic differences between populations may contribute to differences in ADR reporting between countries in a global database of reports of ICSRs. In addition, regional analyses within a global database can serve as an assessment system for pharmacovigilance within a member country. They can be used to specify the performance of pharmacovigilance activities and should be leveraged to catalyse the conversion of the real-world usage into safer use of drugs in ethnically tailored ways.

Supplementary Information The online version contains supplementary material available at https://doi.org/10.1007/s40264-021-01063-1.

Acknowledgements We would like to thank Dr. G. Niklas Norén and Dr. Tetsuo Nakabayashi for their constructive advice and Ms. Eiko Iwasa, Mr. Ryota Kimura and Mr. Yuki Kubota for their excellent assistance. We are also grateful to Dr. Lucie Gattepaille for her support in the revision process, as well as Mrs. Yoko Yoshimoto Tyrefors for her continuous interest in and support of this project. The authors are indebted to the national pharmacovigilance centres that make up the WHO Programme for International Drug Monitoring and contribute reports to VigiBase. The views expressed in this article are the personal views of the authors and may not be understood or quoted as being made on behalf of or reflecting the position of PMDA, WHO or national centres.

\section{Declarations}

Funding Neither Rika Wakao (RW), Ingrid M. Lönnstedt (IL), Yasunori Aoki (YA) nor Rebecca E. Chandler (RC) have received specific grants from any funding agency in the public, commercial, or not-forprofit sectors.

Conflicts of interest/competing interests Neither RW, IL, YA nor RC have no conflicts of interest that are directly relevant to the content of this study. YA is currently employed by AstraZeneca; however, his contributions to this work were made prior to the start of the employment at AstraZeneca, as part of his employment at Uppsala Monitoring Centre and his appointment at the National Institute of Informatics.

Ethics approval Institutional review board approval was not required because VigiBase is an un-linkable anonymized database of WHO.

Consent to participate Consent to participate was not required because VigiBase is an un-linkable anonymized database of WHO.

Consent for publication RW, IL, YA and RC have the support of their respective institutions for publication of this manuscript. All authors read and approved the final version.

Availability of data and material The datasets generated and analysed during the current study are not publicly available due to agreements between contributors of data to the database used (VigiBase) and the custodian of this database. National centres (mainly national drug regulatory authorities) constituting the WHO Programme for International Drug Monitoring (PIDM) contribute data to VigiBase and the Uppsala Monitoring Centre is the custodian in its capacity as the WHO Collaborating Centre for International Drug Monitoring. Some subsets of the data may be available from the corresponding author on reasonable request.

Code availability Code used in the extraction and analysis of data is available upon request.

Authors' contributions RW, RC and IL wrote the manuscript; RW came up with the study concept; RW, RC, IL and YA designed the research; $\mathrm{RC}$ and IL performed the research; IL and YA performed the data analyses and IL contributed analysis methods and graphical overviews. All authors read and approved the final version.

Open Access This article is licensed under a Creative Commons Attribution-NonCommercial 4.0 International License, which permits any non-commercial use, sharing, adaptation, distribution and reproduction in any medium or format, as long as you give appropriate credit to the original author(s) and the source, provide a link to the Creative Commons licence, and indicate if changes were made. The images or other third party material in this article are included in the article's Creative Commons licence, unless indicated otherwise in a credit line to the material. If material is not included in the article's Creative Commons licence and your intended use is not permitted by statutory regulation or exceeds the permitted use, you will need to obtain permission directly from the copyright holder. To view a copy of this licence, visit http://creativecommons.org/licenses/by-nc/4.0/.

\section{References}

1. Sandberg L, Taavola H, Aoki Y, et al. Risk factor considerations in statistical signal detection: using subgroup disproportionality to uncover risk groups for adverse drug reactions in VigiBase. Drug Saf. 2020;43:999-1009.

2. Wakao R, Taavola H, Sandberg L, et al. Data-driven identification of adverse event reporting patterns for Japan in VigiBase, the WHO global database of individual case safety reports. Drug Saf. 2019;12:1487-98.

3. Takano M, Sugiyama T. UGT1A1 polymorphisms in cancer: impact on irinotecan treatment. PGPM. 2017;10:61-8.

4. Zhou Y, Ingelman-Sundberg M, Lauschke VM. Worldwide distribution of cytochrome P450 alleles: a meta-analysis of population-scale sequencing projects. Clin Pharmacol Ther. 2017;102(4):688-700.

5. de Man FM, Goey AKL, van Schaik HN, et al. Individualization of irinotecan treatment: a review of pharmacokinetics, pharmacodynamics, and pharmacogenetics. Clin Pharmacokinet. 2018;57:1229-54.

6. Japanese product label. VFEND. https://www.pmda.go.jp/PmdaS earch/iyakuDetail/ResultDataSetPDF/672212_6179001F1023_3_ 02. Accessed 10 Dec 2020 [Japanese].

7. Japanese product label. Lexapro. https://www.pmda.go.jp/PmdaS earch/iyakuDetail/ResultDataSetPDF/790005_1179054F1022_1_ 16. Accessed 10 Dec 2020 [Japanese].

8. Japanese product label. Plavix. https://www.pmda.go.jp/PmdaS earch/iyakuDetail/ResultDataSetPDF/780069_3399008F1025_1_ 28. Accessed 10 Dec 2020 [Japanese].

9. Lindquist M. VigiBase, the WHO global ICSR database system: basic facts. Drug Inf J. 2008;42(5):409-19.

10. Lagerlund O, Strese S, Fladvad M, et al. WHODrug: a global, validated and updated dictionary for medicinal information. Ther Innov Regul Sci. 2020;54:1116-22.

11. Medical Dictionary for Regulatory Activities. http://www.meddra. org. Accessed 10 Dec 2020. 
12. Norén GN, Orre R, Bate A, et al. Duplicate detection in adverse drug reaction surveillance. Data Min Knowl Discov. 2007;14:305-28.

13. R Core Team R: a language and environment for statistical computing. (2019). https://www.R-project.org/. Accessed 10 Dec 2020.

14. Hopstadius J, Norén GN. Robust discovery of local patterns: subsets and stratification in adverse drug reaction surveillance. In: Proceedings of the 2nd ACM SIGHIT international health informatics symposium. IHI '12. New York, NY, USA: ACM; 2012. pp. 265-74

15. Shiozawa T, Tadokoro J-I, Fujiki T, et al. Risk factors for severe adverse effects and treatment-related deaths in japanese patients treated with irinotecan-based chemotherapy: a postmarketing survey. Jpn J Clin Oncol. 2013;43:483-91.

16. Owusu Obeng A, Egelund EF, Alsultan A, et al. CYP2C19 polymorphisms and therapeutic drug monitoring of voriconazole: are we ready for clinical implementation of pharmacogenomics? Pharmacotherapy. 2014;34:703-18.

17. Medicines \& Healthcare Products Regulatory Agency. Citalopram and escitalopram: QT interval prolongation: New maximum daily dose restrictions (including in elderly patients), contraindications, and warnings. https://www.gov.uk/drug-safety-update/citalopramand-escitalopram-qt-interval-prolongation. Accessed 10 Dec 2020.

18. Clinical Pharmacogenetics Implementation Consortium (CPIC). Guideline for selective serotonin reuptake inhibitors and CYP2D6 and CYP2C19. https://www.cpicpgx.org/guidelines/guideline-forselective-serotonin-reuptake-inhibitors-and-cyp2d6-and-cyp2c 19/. Accessed 10 Dec 2020.

19. Clinical Pharmacogenetics Implementation Consortium (CPIC). Guideline for clopidogrel and CYP2C19. https://cpicpgx.org/ guidelines/guideline-for-clopidogrel-and-cyp2c19/. Accessed 10 Dec 2020.

20. International Council for Harmonisation. E14 guideline. The Clinical Evaluation of QT/QTc Interval Prolongation and Proarrhtyhmic Potential for Nonantiarrhythmic Drugs. https://database. ich.org/sites/default/files/E14_Guideline.pdf. Accessed 10 Dec 2020.

21. Cheng L, Li M, Hu J, et al. UGT1A1*6 polymorphisms are correlated with irinotecan-induced toxicity: a system review and meta-analysis in Asians. Cancer Chemother Pharmacol. 2014;73:551-60.

22. Zhang X, Yin J-F, Zhang J, et al. UGT1A1*6 polymorphisms are correlated with irinotecan-induced neutropenia: a systematic review and meta-analysis. Cancer Chemother Pharmacol. 2017;80:135-49.

23. Kaniwa N, Kurose K, Jinno H, et al. Racial variability in haplotype frequencies of UGT1A1 and glucuroindation activity of a novel single nucleotide polymorphism 686C $>$ T (P229L) found in an African-American. Drug Metab Dispos. 2005;33:458-65.

24. Hikino K, Ozeki T, Koido M, et al. Comparison of effects of UGT1A $1 * 6$ and UGT1A $1 * 28$ on irinotecan-induced adverse reactions in the Japanese population: analysis of the Biobank Japan Project. J Hum Genet. 2019;64:1195-202.

25. Highlights of Prescribing Information. Camptosar. https://www. accessdata.fda.gov/drugsatfda_docs/label/2014/020571s048lbl. pdf. Accessed 10 Dec 2020.

26. Japanese product label. Campto. https://www.pmda.go.jp/PmdaS earch/iyakuDetail/ResultDataSetPDF/800015_4240404A1040_1_ 14. Accessed 10 Dec 2020 [Japanese].

27. Hikino K, Fukunaga K, Mushiroda T. Gap between the US and Japan in coverage of pharmacogenomic biomarkers by health insurance programs: more coverage is needed in Japan. Drug Metab Pharmacokinet. 2018;33:243-9.

28. Moriyama B, Obeng AO, Barbarino J, Penzak SR, Henning SA, et al. Clinical Pharmacogenetics Implementation Consortium (CPIC) Guidelines for CYP2C19 and Voriconazole Therapy. Clin Pharmacol Ther. 2017;102(1):45-51.

29. Kato H, Hagihara M, Hamada Y, et al. Visual disturbance or central symptom like hallucination in patients treated voriconazole: report of six cases. Jpn J Antibiot. 2016;69:143-50.

30. Therapeutic Drug Monitoring guideline. http://www.chemothera py.or.jp/guideline/tdm_es.pdf. Accessed 10 Dec 2020 [Japanese].

31. Levine GN, Jeong Y-H, Goto S, et al. World Heart Federation expert consensus statement on antiplatelet therapy in East Asian patients with ACS or undergoing PCI. Nat Rev Cardiol. 2014;11:597-606.

32. Highlights of Prescribing Information. Effient. https://www.acces sdata.fda.gov/drugsatfda_docs/label/2010/022307s002lbl.pdf. Accessed 10 Dec 2020.

33. Japanese product label. Efient. https://www.pmda.go.jp/PmdaS earch/iyakuDetail/ResultDataSetPDF/430574_3399009F1020_1_ 17. Accessed 10 Dec 2020 [Japanese].

34. Yoon HY, Lee N, Seong J, et al. Efficacy and safety of clopidogrel versus prasugrel and ticagrelor for coronary artery disease treatment in patients with CYP2C19 LoF alleles: a systemic review and meta-analysis. Br J Clin Pharmacol. 2020;86:1489-98. 